\title{
Deep infiltrating endometriosis: role of magnetic resonance subtraction imaging
}

\author{
Darshan Gandhi $^{1}$, Gunjan Garg ${ }^{2}$, Shantanu Solanki ${ }^{3}$, Pankaj Nepal ${ }^{1}$ \\ ${ }^{1}$ St. Vincent's Medical Center, Bridgeport, CT, USA; ${ }^{2}$ Yale New Haven Health System, Bridgeport Hospital, Bridgeport, CT, USA; ${ }^{3}$ Guthrie Robert \\ Packer Hospital, Sayre, PA, USA
}

Correspondence to: Darshan Gandhi. St. Vincent’s Medical Center, Bridgeport, CT, USA. Email: darshangandhi7@gmail.com.

Submitted Aug 07, 2018. Accepted for publication Aug 15, 2018.

doi: 10.21037/qims.2018.08.06

View this article at: http://dx.doi.org/10.21037/qims.2018.08.06

We read the manuscript titled "Deep infiltrating endometriosis MR imaging with surgical correlation" by Tang et al. with educational interest (1). We would like to congratulate the authors for presenting valuable pictorial review of deep infiltrating endometriosis (DIE) with meticulous description of magnetic resonance imaging (MRI) findings of endometriosis. The authors described imaging findings of DIE like-T1 hyperintensity on T1 fat saturated images, "T2 shading sign", restricted diffusion in the lesion with imaging findings in high and low $\mathrm{b}$ values and contrast enhancement in the lesion with fat suppressed post contrast T1 imaging $(1,2)$; however, we stress on the crucial role of MR subtraction imaging in diagnosing endometriosis, particularly small deep pelvic implants (3). Subtraction imaging utilizes fat saturated $\mathrm{T} 1$ non-contrast and fat saturated $\mathrm{T} 1$ post gadolinium contrast sequences using identical imaging criteria. Another image series is created using digital subtraction technique and hence remaining signal in subtraction is merely attributed to enhancement $(3,4)$. It is particularly helpful if the deep infiltrating endometriotic lesion is adjacent to or within the hemorrhagic or proteinaceous fluid in cul-de-sac where T1 imaging shows hyperintensity regardless of gadolinium contrast, thus subtraction sequence solely shows enhancing foci of endometriosis, clinching the diagnosis (3). This feature of subtraction imaging is moreover valuable in DIE where lesion itself is hyperintense to muscle in T1 noncontrast sequences and qualitative evaluation of contrast enhancement is challenging in contrast enhanced T1 fat saturated sequence $(1,2,4)$. We came across a 36 -year-old female with pelvic pain and a clinical diagnosis related to endometriosis. Pelvic MRI with subtraction imaging sequence revealed deep pelvic endometriotic implants (Figure 1). We do recommend performing conventional post contrast $\mathrm{T} 1$ weighted fat saturated imaging, however, subtraction MRI can be extremely useful in differentiating complex T1 hyperintense cystic lesions like, ectopic pregnancy where contrast enhancement may be mild and with subtraction images, differentiation will be much clearer from endometriosis (5). Role of subtraction imaging is crucial in unusual presentation of endometriosis including but not limited to scar endometriosis, DIE, endometrioma with infection or endometrioma in adenomyotic uterus (6). Thus, this letter is written with the aim that readers can appreciate the proposed plan for patients with DIE undergoing MRI. 

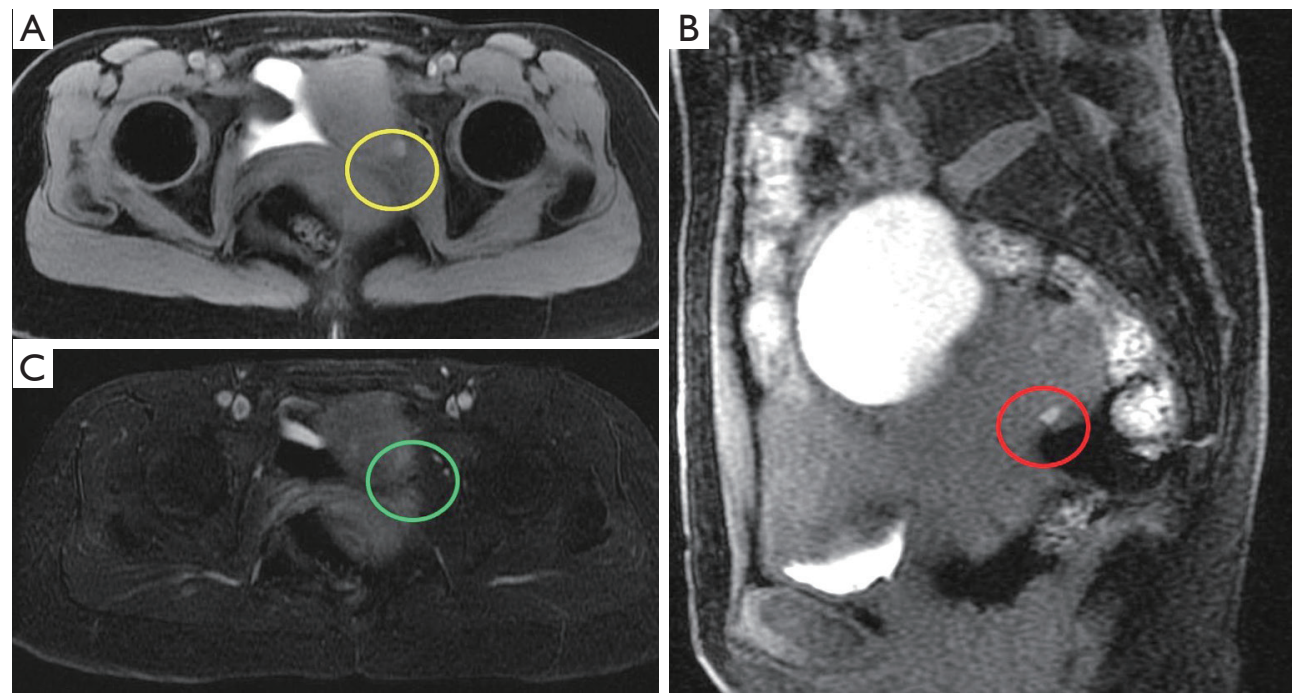

Figure 1 Axial T1 weighted post gadolinium contrast image of the pelvis (A) shows a T1 hyperintense focus in the left hemipelvis (yellow oval). Sagittal T1 weighted image (B) confirm the T1 hyperintense deep left pelvic endometriotic implant (red oval). Axial post gadolinium contrast T1 weighted subtraction image (C) shows no enhancement in the deep pelvic endometriotic implant (green oval).

\section{Acknowledgements}

None.

\section{Footnote}

Conflicts of Interest: The authors have no conflicts of interest to declare.

\section{References}

1. Tang X, Ling R, Gong J, Mei D, Luo Y, Li M, Xu J, Ma L. Deep infiltrating endometriosis MR imaging with surgical correlation. Quant Imaging Med Surg 2018;8:187-95.

2. Siegelman ES, Oliver ER. MR imaging of endometriosis:

Cite this article as: Gandhi D, Garg G, Solanki S, Nepal P. Deep infiltrating endometriosis: role of magnetic resonance subtraction imaging. Quant Imaging Med Surg 2018;8(7):722723. doi: 10.21037/qims.2018.08.06 ten imaging pearls. Radiographics 2012;32:1675-91.

3. Newatia A, Khatri G, Friedman B, Hines J. Subtraction imaging: applications for nonvascular abdominal MRI. AJR Am J Roentgenol 2007;188:1018-25.

4. Eid M, Abougabal A. Subtraction images: A really helpful tool in non-vascular MRI. The Egyptian Journal of Radiology and Nuclear Medicine 2014;45:909-19.

5. Chanana C, Gupta N, Bansal I, Hooda K, Sharma P, Gupta M, Gandhi D, Kumar Y. Different Sonographic Faces of Ectopic Pregnancy. J Clin Imaging Sci 2017;7:6.

6. Bennett GL, Slywotzky CM, Cantera M, Hecht EM. Unusual manifestations and complications of endometriosis--spectrum of imaging findings: pictorial review. AJR Am J Roentgenol 2010;194:WS34-46. 